almost any sort of protein. For example, the serum of an animal immunized against bovine serum will produce precipitins that react only with bovine serum and not at all with any other proteins; but if bovine serum is first saturated with iodin before being used for immunization, the serum of the injected animal will react not only with bovine serum but also with horse serum, human serum, milk, or any other of a large list of different protein containing fluids. Of similar import is Fleischmann's observation that tryptic digestion of serum likewise causes the serum to be capable of producing only non-specific precipitins; for in tryptic digestion it is the tyrosin and tryptophan radicals that are first split out of the protein molecule. Vaughan and his pupils have been able to split proteins of various sorts into toxic and non-toxic portions, and at the recent meeting of the Association for the Advancement of Seience, Dr. Vaughan stated that the toxic portion of the protein molecule contains the aromatic radicals, and that gelatin, which is notoriously poor in aromatic radicals, does not yield a toxic portion.

The connection between the facts mentioned above and the remarkalule phenomenon of hypersensitization or anaphylaxis, seems apparent, and it was thought that if these observations and deductions are correct, then the toxic effect of a second dose of a protein injected into an animal that has been sensitized by a previous injection of the same sort of protein given two weeks or more previously, may be dependent on the aromatic radicals of the proteins. To test this hypothesis severas attempts have been made, with various modifications of dosage, to obtain sensitization of guinea-pigs to gelatin, which contains in the way of aromatic radicals only a small quantity of phenyl alanin, no tryptuphan, and most minute or no traces of tyrosin. In no case has scnsitization been obtained, although, so far as the literature shows, every other form of protein that has yet been investigated will sensitize guinea-pigs. These results are in agreement with Vaughan's fincing that no toxic group can be split out of the gelatin molccule, and indicate that the toxic substance concerned in the anaphylaxis reaction in some way depends on the aromatic radicals of the proteins, just as the specificity of the precipitin reaction seems to be due to these same radicals. As Anderson and Rosenau ${ }^{1}$ have suggested in their valuable studies, this reaction of anaphylaxis seems to be fundamentally related to many of the vital principles of infection and immunity, and if further studies that are under way can establish what the gelatin experiments indicate, that this reaction depends on certain specific groups of the protein molecule, an interesting line of rescarch into the chemistry of these reactions will be opened.

\section{FULMINATING PELVIC-ABDOMINAL EDEMA SIMULATING RUPTURED TUBAL PREGNANCY.}

\section{WALLACE A. BRIGGS, M.D. SACRAMENTO, CAL.}

The similarity of the symptoms to those of ruptured tubal pregnancy in two cases of fulminating pelvicabdominal edema which came under my observation led me to search the literature for analogous cases. Extensive research and careful study of cases revealed only two cases of fulminating pelvic-abdominal edema in

1. Jour. Infect. Dis., 1908, $\mathbf{7}, \mathbf{8 5 .}$ which a diagnosis of ruptured tubal pregnancy had been made. In one of these the diagnosis of retro-uterine hematocele was changed to salpingitis with suppurative ovaritis before operation was decided on. For purposes of comparison it might be well to give a brief history of these cases before citing my own:

JOCET'S CASE.-The first case was reported by Jocet in 1897 . It occurred in a woman aged 28 , who had been married eight rears; no children. There was a history of having experienced on two different occasions severe pelvic-abdominal pain accompanied by accumulation of fluid in the right iliac fossa, which presented the characteristics of hematocele. These tumor's terminated in resolution and the patient was entirely well during the intervals. She had a third attack in September, 1907, which was again diagnosed by another physician as hematocele and, in the light of the previous history, treated expectantly. Her condition improved, but on November 23 she was suddenly attacked by violent abdominal pains and almost incessant vomiting, accompanied by pallor, anxious facies, dry and flaceid skin. At the time of examination, chill; abdomen slightly tympanitic. A large fluctuating mass in the right iliac fossa extended within a hand's breadth of the crural arch. Right lateral cul-de-sac presented a tumefaction which appeared continuous with that of the iliac fossa. Bouilly positively excluded hematocele and diagnosed the condition as salpingitis, with suppurative ovaritis or inflamed evstic ovary. Operation November 25. Incision in right posterolateral cul-de-sac was followed by a diseharge of a large quantity of perfectly limpid fluid. A fluctuating cyst could now be palpated in the abdomen and Bouilly, believing this to be en ovarian cyst which had given rise to the peritoneal phenomena, performed laparotomy and exposed a large gelatiniform, encysted peritoneal mass in the iliac fossa, filled with a clear fluid. Underneath the peritoneal edema was a whitishpink layer which formed the wall of the true sac. This was punctured and a considerable quantity of fetid pus evacuated. The pus sac was found to be the greatly distended suppurative ovary; it was separated from adhesions and enucleated. Recovery uneventful.

LEGUEU'S CASE.-The second case was observed by Legueu in 1901. Female, aged 21, was admitted to Hospital Hotel Dieu, May 16, 1901. Menstruation began at age of 16 and had always been regular. She aborted in May, 1900, and complained of pelvic disturbances since then. About fifteen days preceding date of admission to hospital, after regular and normal menstruation, she was suddenly attacked by violent pelvic pain accompanied by syncope, extreme pallor, cold extremeties, etc. The abdomen was enlarged. Pain diminished somewhat, but the abdomen remained distended, hard and painful to pressure. Vaginal examination disclosed a fluctuating mass in the posterior cul-de-sac. A diagnosis of retrouterine hematocele was made. On opening the abdomen a quantity of yellow, serous fluid escaped. It was found that a serous exudate had developed around the right adnexa. This contained 500 grams of fluid and extended from the cul-de-sac of Douglas below to a wall of its own above, formed of a fibrinous network of tissue. The adnexa were removed and the roof of the serous cavity allowed to remain. Recovery was accompanied by slight postoperative suppuration. The serous fluid was examined and found to contain leucocytes and desquamated peritoneal epithelial cell.

Briggs' CASES.-I will now report my own observations:

CASE 1.-Mrs. G., ${ }^{1}$ came to my office complaining of malaise and pelvic pain. Her menstruation had been normal. By a pelvic examination I discovered only a slight and obscure fulness about the left adnexa. The uterus was normal in size and consistency. Neither the history nor the local condition suggested ectopic pregnancy. Two days later the patient again came to my office. Her face was pale, anxious, pinched; her pulse 120 , small and weak; temperature $100 \mathrm{~F}$. The pelvis was completely filled with a fluctuating mass. Notwithstanding the early history I made a diagnosis of ruptured tubal pregnancy. On opening the abdomen $I$ found the pelvis en-

1. Case reported to the Occidental Medical Times, Author give date- with a series of twenty-one ectopic pregnancies. 
tirely roofed over and when the agglutinated surfaces were separated scrum poured out of them like sap from succulent bark in the spring time. Convinced that the case was one of fulminant pelvic peritonitis instead of misplaced pregnancy, and fearing an almost certain infection of the general peritoneum if the operation were completed from above, I closed the abdomen and entered the pelvic peritoneum through the posterior fornix. About a pint of serum was evacuated and gauze drainage thoroughly established. Uneventful recovery.

Case 2.-Mrs. - aged 34 vears, has had two full term pregnancies and six abortions; has had more or less discomfort for several years, which, however, did not impair either her general health or her capacity for work and for which she did not consult a physician. Her last menstruation appeared Jan. 21, 1905, four days overdue; it was scanty and intermit tent but not otherwise abnormal until January 28, when I was called. I found the patient suffering with rather severe paroxysmal pelvic pain, which she ascribed to abortion. Pulse and temperature were normal; the general condition was excellent; flow moderate and bright. I ordered codein and phenacetin to relieve pain.

January 30,2 p. m., patient has had severe pelvic pain extending into the abdomen, particularly on the right, since $8 \mathrm{p.} \mathrm{m}$. yesterday; has had two "fainting spells;" temperature normal; pulse 90, small and compressible; abdomen sensitive, particularly near the pelvic brim, but soft and pliable. Gave $1 / 4 \mathrm{gr}$. of morphin hypodermically and ordered it repeated every hour by mouth until pain was relieved. At $6 \mathrm{p}$. m. patient was relieved, but evidence of shock was more pronounced; radial pulse imperceptible; skin and mucous membrane were markedly anemic; uterus retroverted, enlarged, fixed, sensitive; adnexa indistinguishable; liquid could be demonstrated in the flanks on either side but insufficient in quantity to transmit a wave. Feeling that the case was one of shock due to internal hemorrhage arising from ruptured tubal pregnancy, I advised an immediate laparotomy. At once the patient's face flushed a deep scarlet and the diagnosis, which a moment before I regarded as practically positive, was open to serious question. The deep flush seemed irreconcilable with the theory of so grave an internal hemorrhage. The doubt was fully admitted, but exploratory incision was urged as the only means of diagnosis and possible treatment. A hypodermic of adrenalin and a high enema of saline solution were administered and the patient was taken to the hospital. Exploratory incision at 10:30 p. m. Preperitoneal connective tissues filled with great blubbers of serum which extended to the level of the umbiliens, confusing the relation of parts and necessitating a prolongation of the incision upward. Three pints of serum were taken from the abdominal eavity; the pelvic peritoneum pouched into the pelvic cavity from every direction; the left tube was chronically inflamed, the right tube imbedded in adhesions; what seemed to be the right ovary was as large as a goose egg and crstic. The abdomen was closed without further molestation. Consciousness returned but the patient did not rally and died at $2: 30$ a. m.

Autopsy by Dr. Tuitchell.-Three pints of serum in peritoneal cavity; connective tissue as high up as umbilicus anteri. oriy and the diaphragm posteriorly filled with serum; mesentery filled with serum; pelvic peritoneum pouching into pelvic cavity from every direction and presenting the appearance of cysts; what at the operation seemed to be the cystic right ovary proved to be a peritoneal bag filled with serum; rioht ovary and tube imbedded in recent adhesions, right tube thickened but free from pus, left tube slightly thickened and injected but free; with the exception of that covering the right tube and ovary the peritoneum nowhere presented evidence of inflammation.

These two cases, as well as the case observed by Leguen, have much in common, both symptomatically and pathologically: symptomatically a close simulation of ruptured tubal pregnancy; pathologically the rapid accumulation of a large quantity of serum both subperitoneally and intraperitoneally, in one case positively, in the other probably, beginning in the pelvis and ex- tending upward, in the earlier case to the false pelvis, in the latter to the diaphragm. In one case certainly, in the other case probably, there was a nidus of inflammation in the tube. Unfortunately Legueu does not state at what point the limiting fibrinous membranes had formed, nor does he give any information concerning the pathologic condition of the right adnexa which he removed.

Both of my cases so closely simulated ruptured tubal pregnancy that a positive diagnosis was prevented in the former case only by the absence of the usual history preliminary to rupture, and in the later case only by the sudden deep flushing of the face inconsistent with exsanguination. In the later case a confident diagnosis was expressed and I believe would have been expressed by the most experienced diagnostician before the flush described in the case report. The menstruation, hemorrhagic in character and delayed a week, the pelvic findings, the excruciating pain radiating from the pelvis, the seeming acute anemia with evident shock increasing to collapse, afforded grounds for a positive diagnosis of ruptured tubal pregnancy. The deep flush of the face following excitement was incompatible with this diagnosis.

Toward an etiologic solution of these cases $I$ confess my inability to contribute anything positive. In botl cases there was a mild inflammatory condition, in the earlier case producing agglutination of the peritoneli. I and roofing in the pelvis, and in the later case confined to the right adnexa. In the second case particularly angioneurotic edema suggests itself as a not improbable solution. The patient was highly neurotic and for a year or more preceding the fatal attack, beside doing her own housework, sewing, etc., had given a great deal of time to preparing herself for a musical career. Besides these there were other demands on her nervous sustem which may have contributed to the neurasthenic condition.

\section{CONCLUSIONS.}

1. Fulminating pelvic edema may very closely simulate a ruptured tubal pregnancy.

2. Absence of subjective and objective signs of pregnancy, of irregular uterine hemorrhage, of decidua in the flow in cases of shock with severe pelvic pain, would suggest the possibility of fulminating pelvic edema.

3. A hemoglobin test might assist in the differential diagnosis.

4. Fulminating pelvic edema is probably angioneurotic in origin.

5. Death (as in Case 2) may result from acute pelvicabdominal edema.

Glaucoma and General Affections.-Blessig of St. Petersburg warns that glaucoma should not be regarded as an independent and purely local affection, but rather as a manifestation of some general trouble. It is generally a symptom of pathologie changes in the vascular system. Reduction of the pressure in the heart arteries and corresponding increase in the venous pressure may suffice to transform a merely glaucomatous tendency into actual glaucoma. Zimmermann has reported great benefit from heart tonics, especially strophanthus, to reduce the difference between the general blood pressure and that within the eye. Emotional stress and neuralgia may also start or aggravate a glaucomatous condition. Hirth found sclerosis of the carotids in 46 among 69 glaucomatous patients. The specialist and the internist must work hand in hand, Blessig concludes, in order to control glaucoma. His article was published in the St. Petersb. med. Wochschr., Sept. $7,1907$. 